\title{
investigación
}

\section{MORTEROS COLA}

\section{Características y condiciones de empleo}

\author{
César del Olmo, Angel Ruiz de Gauna, \\ Antonio Ruiz Duerto y Bernardo Torroja* \\ IETcc \\ $679-10$
}

\section{Resumen}

El presente trabajo recoge algunos de los aspectos científicos y técnicos relativos a la utilización de los morteros cola, para la ejecución de revestimientos cerámicos, con el fin de facilitar al lector un mejor conocimiento de sus características $y$ de sus posibilidades de empleo. El trabajo se ha estructurado de la siguiente forma: 1.-Antecedentes. 2.-Definición y aplicación. 3.-Composición. 4.-Mecanismo de actuación. 5.-Comportamiento reológico. 6. - La unión adhesiva. 7.-Propiedades de los morteros cola. 8.-Modo de medir estas características. 9.-Bibliografía.

\section{Antecedentes}

Desde antiguo, a los materiales de construcción tradicionales (mortero de cal, yeso, etc.) se les han incorporado para usos específicos ciertas sustancias, conocidas con el nombre de aditivos - añadidas en pequeñas proporcionescon el fin de proporcionar, modificar o incrementar en el material tradicional determinadas características.

Una mejora en las propiedades como material de agarre, a veces notable, se ha logrado con la incorporación de sustancias naturales, tales como: arcilla, albúmina de sangre, colas de origen animal, almidón, etc., que constituyen -al igual que puede ser considerado el propio cementosustancias poliméricas.

\section{Definición y aplicación}

Se conoce con el nombre de mortero cola un mortero mixto de cemento y resina, fabricado en planta industrial,

\footnotetext{
* Miembros del Equipo de Investigación para el Estudio y Desarrollo de Nuevos Materiales para Revestimientos Continuos.
}

que se emplea para la colocación (encolado) de piezas cerámicas como revestimiento, tanto de paredes como de suelos.

Se trata de morteros cuyos componentes se encuentran dosificados en seco, y se suministran envasados con marca de fabricación.

El producto se prepara para su uso agregando simplemente la cantidad de agua suficiente para proporcionar a la mezcla resultante la consistencia adecuada.

La aplicación de la pasta así obtenida se realiza en capa fina, generalmente extendiendo ésta sobre el soporte con llana y haciendo pasar por ella, a continuación, una espátula dentada (peinado del producto) cuyo objeto es garantizar la uniformidad de su espesor. Aunque el material también puede ser aplicado en la parte posterior de la loseta, extendiendo una pequeña cantidad de pasta en la zona central, o cantidades menores en zonas más puntuales separadas entre sí (aplicaciones que se denominan en masa o por puntos, respectivamente), esta práctica sólo es admisible para revestimientos interiores no sometidos a la acción directa del agua.

El mortero cola representa una mejora notable de las propiedades de los morteros tradicionales, no sólo en lo que se refiere a las condiciones de puesta en cbra (grado de adherencia inicial alcanzado durante la misma - pegajosidad - y al poder de retención del agua de amasado), sino en cuanto a sus resultados finales (resistencia a cizallamiento, flexibilidad).

Se define como "pegajosidad» en la tecnología de los adhesivos, la adherencia «instantánea» de un material al contacto inmediato con otra superficie; el valor de esta adherencia inicial difiere de la adherencia final que el producto alcanza después de curado.

Se puede conseguir también en el mortero tradicional una cierta mejora de estas dos propiedades, sustituyendo una pequeña proporción en peso del árido (arena) del mismo -entre un $5 \%$ y un $15 \%$ por una proporción equivalente de arcilla; sin embargo, este incremento de la pegajosidad se logra a costa de una reducción importante de las resistencias mecánicas del material.

La incorporación de arcilla indicada hace que el mortero requiera, para conseguir la misma consistencia, un aumento de la proporción del agua de amasado. Esta mayor cantidad de agua incorporada al material produce dos hechos de signo contrario: por una parte, origina la reducción mencionada de las resistencias mecánicas del material, como consecuencia de la disminución de las fuerzas de cohesión que en el mismo da lugar el incremento de huecos (poros) que deja el exceso de agua añadida, al ser eliminada.

Pero también, esta mayor cantidad de agua embebida en el material (almacenada en las micelas del gel de 
arcilla), permite que el mortero de agarre, en general, conserve una proporción de agua suficiente para conseguir la prácticamente total hidratación del cemento, a pesar de la pérdida de agua que supone el contacto del mismo en obra con el soporte poroso y con la plaqueta cerámica a colocar (12).

El producto está pensado - proyectado- para una puesta en obra de tipo industrializado permitiendo con su utilización alcanzar, en las operaciones de alicatado y solado, una gran economía de tiempo y de rendimiento en la mano de obra, para lo cual las dos propiedades antes mencionadas de elevada adherencia inicial y poder de retención de agua, son de capital importancia. La primera, porque permite la colocación del revestimiento cerámico sobre soportes lisos (sin el picado previo del soporte como en el caso de superficies de hormigón, por ejemplo) así como corregir la posición de las piezas; y la segunda, porque libera al operario de tener que humedecer el soporte y la pieza a colocar, operaciones imprescindibles de realizar con un mortero de agarre tradicional.

Este tipo de aplicación industrializada no propicia, en general, que el material de agarre pueda desarrollar la máxima potencialidad de adherencia de que es capaz por sus características intrínsecas, dado que la filosofía que con frecuencia rige este tipo de puesta en obra estriba en lograr la máxima velocidad de colocación, aun conservando un mínimo de calidad en la unión adhesiva la cual es superior, en general, a la conseguida con los morteros tradicionales. Sin embargo, tales características de máxima adherencia final pueden conseguirse con una aplicación del mortero cola más cuidada.

Por la razón expuesta el material, si es de buena calidad, está dotado de un alto poder adhesivo - que sólo se utiliza parcialmente en dicha aplicación industrializada-, de tal manera que la potencial fuerza de adherencia final del producto, tanto al soporte (mortero, cerámica, hormigón, etc.) sobre el que se aplica, como a la pieza cerámica que sujeta, supera con frecuencia a las fuerzas de cohesión que unen entre sí la propia masa de estos materiales por lo que, en caso de arrancamiento, la rotura del conjunto soporte/pieza cerámica se produce muchas veces por la masa del soporte o, con más frecuencia, por la propia pieza cerámica y no por la superficie de contacto - unión - de uno y otro con el material de agarre.

\section{Aunque, desde el punto de vista práctico, la} preocupación principal de cualquier unión adhesiva radica en la fuerza total de la unión y no en donde se produce el fallo.

El mortero cola es un material que constituye una modalidad de sujeción de piezas cerámicas intermedia entre las que representan: el mortero de agarre tradicional, y las nuevas colas y pastas preparadas para empleo inmediato, introducidas no hace mucho en el mercado europeo y americano de la construcción con destino a la edificación industrializada. Estos tres tipos de materiales de sujeción responden a otras tantas etapas de la edificación, en que se trabaja con reducciones crecientes en las tolerancias de planeidad en paramentos y suelos (soportes) donde se colocan los acabados superficiales mencionados.

\section{Composición}

En resumen, la formulación de los morteros cola está compuesta por un mortero de cemento modificado con macromoléculas orgánicas (resinas), de origen natural y/o sintético (caseínas, polímeros vinílicos, derivados de celulosa, etc.).

Los primeros morteros cola fabricados en planta industrial estaban formados por un mortero de cemento y arena silícea con granulometría fina (tamaño máximo del árido comprendido aproximadamente entre 0,1 y $0.5 \mathrm{~mm}$ ), al que se incorporaba una pequeña proporción de adhesivo orgánico del tipo caseína.

Como complemento de la caseína, en general, se incorporaba además un producto como regulador de la alcalinidad $(\mathrm{pH})$, constituido con frecuencia por bórax y añadido en pequeñas proporciones.

Posteriormente se prepararon productos de mejores características globales existiendo en el mercado español una amplia oferta autóctona de estos materiales, de alta cualificación, cuyas características y prestaciones estaban de conformidad con las directrices adoptadas por la casi totalidad de los países europeos y emanadas de la Union Européenne pour l'Agrément Technique dans la Construction -U.E.A.t.c. - (Unión Europea para la Apreciación Técnica en la Construcción).

\section{En posesión muchos de ellos del Documento de Idoneidad Técnica (D.I.T.) para materiales y procedimientos de construcción no tradicionales.}

La composición clásica de estos materiales era igualmente un mortero de cemento y arena silícea, al que se añadian adhesivos orgánicos formados, por lo general, por una resina vinícola y otra celulósica.

\section{Más concretamente las resinas vinílicas están constituidas, por lo general, por acetato de polivinilo; y las resinas celulósicas, por éteres de celulosa.}

La formulación media estimada de estos productos, tomando como referencia los Documentos de Idoneidad Técnica franceses, es la siguiente:

$$
\begin{aligned}
& \text { - Cemento portland } \\
& \text { - Arena silícea .. } \\
& \text { - Constituyentes secundario................. } \\
& \text { vinílicas y celulósicas)... }
\end{aligned}
$$

\section{Cahiers del CSTB.}

No obstante, en la actualidad, se advierte en nuestro país un descenso bastante generalizado de la calidad de estos materiales de agarre, como consecuencia de que la respuesta - demanda - del mercado a la calidad of recida por los fabricantes no ha sido la adecuada. La composición de estos morteros cola está formada igualmente, en general, por un mortero de cemento y arena silicea, pero la proporción de las resinas vinílicas $y$ celulósicas -que constituyen los productos de mayor precio dentro de la formulación - ha disminuido, habiéndose eliminado además del material, en algunos casos, el primero de los dos adhesivos mencionados.

\section{Mecanismo de actuación}

El funcionamiento del mortero cola responde a un mecanismo complejo, no bien determinado, que es la resultante de las actuaciones individuales de los 
constituyentes activos del mismo (cemento y macromoléculas orgánicas), modificadas estas actuaciones de forma importante por interacciones entre estos productos.

El grado de adherencia final del mortero cola depende también de la historia de curado del mismo, además de los aspectos relativos a las características de los elementos a unir (soporte/pieza cerámica) y de la forma de su puesta en obra, que se analizan más adelante (apartado 6).

El mortero cola se caracteriza porque los constituyentes activos que contiene necesitan condiciones completamente distintas para su curado y endurecimiento. Los aditivos orgánicos endurecen por secado como consecuencia de la eliminación del agua interpuesta entre las macromoléculas, y de la subsiguiente unión de las macromoléculas entre sí por fuerzas fisicoquímicas, mientras que el cemento endurece por reacción entre las moléculas anhidras del mismo con el agua y da lugar a la formación de nuevos compuestos cristalinos (hidratos), que se entrelazan y traban dando un conjunto resistente.

Al mezclarse el producto en polvo con el agua de amasado se forma un sistema de tres fases (sólido granulado-liquido-aire). Los polimeros presentes en el material forman una dispersión coloidal que, por las caracteristicas de actividad superficial, tienden a interponerse entre las partículas de cemento «lubrificando» el contacto entre las partículas sólidas y, en consecuencia, modificando la plasticidad de la pasta preparada. De esta mayor plasticidad y de la actividad superficial del polímero depende, por lo general, la adherencia inicial del producto.

Como consecuencia de la actividad superficial del polímero, se deposita sobre los granos de cemento una película de polimero que les cubre parcialmente (en mayor o menor grado según sea la proporción de aditivos orgánicos incorporados en relación con el peso del cemento). Esta película impide que progrese la hidratación del cemento, por lo que el fraguado normal del mismo se retrasa.

El curado de la pasta de mortero cola se hace en seco, por esta razón el cemento del mismo no llega a hidratarse en su totalidad, permaneciendo una parte como material inerte; sin contribuir, por lo tanto,

a las resistencias mecánicas del material de agarre.

Cuando la pasta endurecida de mortero cola se pone en contacto en etapas posteriores, con la humedad, se producen dos hechos de signo contrario. Por una parte, el agua produce en el material, mientras la humedad permanece en el mismo, un aumento de volumen (hinchamiento), como consecuencia de que el agua absorbida se interpone entre los enlaces de Van der Waals del polimero, originando una disminución apreciable de las características mecánicas de adherencia y cohesión del material, y, al mismo tiempo, produce también una hidratación de las fracciones del cemento no hidratadas.

Tras el secado, el mortero cola recupera, por lo general, no sólo la caída de resistencias mecánicas de adherencia indicadas (originadas por efecto del hinchamiento mencionado), sino que incluso se detecta en el mismo un aumento de la adherencia, debido a la hidratación de las fracciones de cemento no hidratadas previamente.

\subsection{Influencia en las Características Tecnológicas}

La cinética (velocidad) de las reacciones quimicas y fisicoquímicas en que intervienen los constituyentes activos del mortero cola y el modo cómo avanzan estas reacciones, condiciona, en particular, el valor de determinados parámetros del material, que sirven para definir ciertos aspectos del comportamiento tecnológico del mismo. Nos referimos en concreto a los denominados: Tiempo de vida de la pasta en el bote (Pot life), Tiempo abierto (o de unión) y Tiempo de ajustabilidad manual.

Para que la pasta del mortero cola adquiera unas propiedades de adherencia mínimas, tanto inicial como final, se requiere que transcurra un tiempo - de esperamínimo, desde la mezcla del producto en polvo con el agua, para dar oportunidad a que se desarrollen las reacciones que tienen lugar en los constituyentes activos del material (principalmente el paso de los polímeros orgánicos a disolución coloidal y las primeras etapas de la hidratación del cemento).

Por lo general, este tiempo mínimo de espera es de unos 30 min., superior al mínimo de 15 ó $20 \mathrm{~min}$. recomendado por los fabricantes del material.

También la pasta preparada tiene una vida limitada, hecho que está condicionado principalmente porque el fraguado del cemento (avance de las reacciones de hidratación), limita el tiempo de puesta en obra del material y disminuye sus características adherentes.

Al producto se le exige un tiempo de vida mínima en el bote de 3 horas, limitación que supera con facilidad, debido a que el fraguado normal del cemento se retrasa con la incorporación de estos aditivos orgánicos.

El Tiempo abierto se refiere al lapso de tiempo durante el cual, una vez expandida sobre el soporte una capa de la pasta de mortero cola y peinada ésta, es posible mantener sobre aquél por medio de ellas las piezas a colocar y alcanzar una adherencia final suficiente (igual o superior a $5 \mathrm{~kg} / \mathrm{cm}^{2}$ ).

Después de extendida la pasta de mortero cola sobre el soporte se produce un reflujo paulatino, hacia la superficie del material, de una parte de los aditivos orgánicos contenidos en el mismo, junto con las burbujas de aire ocluidas por éstos en la pasta; hecho que se aprecia con facilidad por la formación de una capa superficial de color blancuzco brillante de pequeño espesor. El tiempo transcurrido entre el extendido de la pasta y la aparición de la película superficial se llama «tiempo de formación de película», el cual depende de la formulación del producto y tiene una gran influencia, a efectos de las condiciones de puesta en obra, y para la durabilidad del revestimiento (ver Apartado 6.3).

La formación de la película indicada hace que se alteren las fuerzas de cohesión inicial de la pasta, reduciéndose el poder global de adherencia de la misma y con ello el Tiempo abierto del mortero cola.

Por lo general, el tiempo abierto de estos materiales varia en condiciones de laboratorio entre 20 y $30 \mathrm{~min}$., quedando reducido este parámetro de forma importante a medida que disminuye en la formulación del material el contenido de polímeros. 
El Tiempo abierto - válido - se considera finalizado cuando el valor de la adherencia obtenido en el ensayo cae por debajo de $5 \mathrm{~kg} / \mathrm{cm}^{2}$.

Por último, se conoce con el nombre de Tiempo de ajustabilidad manual, al lapso de tiempo durante el cual, después de sujeta una pieza al soporte con el mortero cola, es posible rectificar con movimientos exclusivos de rotación la posición de la misma, ya colocada, sin pérdida sensible de la adherencia final al soporte.

El tiempo de ajustabilidad se considera igualmente finalizado cuando el valor de la adherencia, obtenido en el ensayo, cae por debajo de $5 \mathrm{~kg} / \mathrm{cm}^{2}$.

El ajuste manual de la pieza suele producir la ruptura de la película superficial que pudiera haberse formado, por lo que el valor de la adherencia obtenido en el ensayo es superior a los valores que resultan en el ensayo de tiempo abierto a edades iguales (donde, por la técnica operatoria aplicada, no se produce la ruptura de dicha película).

\section{Comportamiento reológico}

Las suspensiones fuertemente concentradas como son las pastas de mortero cola, pueden ser consideradas, por lo que concierne a su comportamiento reológico, como cuerpos de Bingham.

Se conoce con el nombre de reología la ciencia que estudia el flujo y deformación de la materia.

Se dice que un cuerpo es del tipo Bingham o plástico cuando el gradiente de la velocidad de deformación del material $\left(\frac{\mathrm{dv}}{\mathrm{dz}}\right)$ que aparece cuando se le aplica un esfuerzo cortante $\mathrm{F}$, es proporcional a este esfuerzo más allá de un cierto umbral f.

$\mathrm{F}-\mathrm{f}=\eta \quad\left(\frac{\mathrm{dv}}{\mathrm{dz}}\right) \cdot \mathrm{S}$

Donde $f$ es el umbral de cizallamiento o esfuerzo mínimo de flujo o tracción crítica;

$\eta$ la viscosidad; $y$

$\mathrm{S}$ la superficie del plano en movimiento.

Pero más concretamente, en los morteros cola se favorece que su actuación reológica presente una ligera tixotropía.

Tixotropía es la propiedad física en virtud de la cual una suspensión se licua (y desliza) bajo el efecto de una fuerza aplicada sobre ella (para el extendido de la pasta sobre el soporte, para la agitación de la misma en el bote, etc.) y se vuelve rígida en reposo.

La figura 1 contiene una visión panorámica de los diagramas reológicos de los fluidos, que puede servir para encuadrar los cuerpos de Bingham y tixotrópicos aludidos.

\section{La unión adhesiva}

En primer lugar, cabe recordar que se entiende por adherencia de un mortero la capacidad del material de

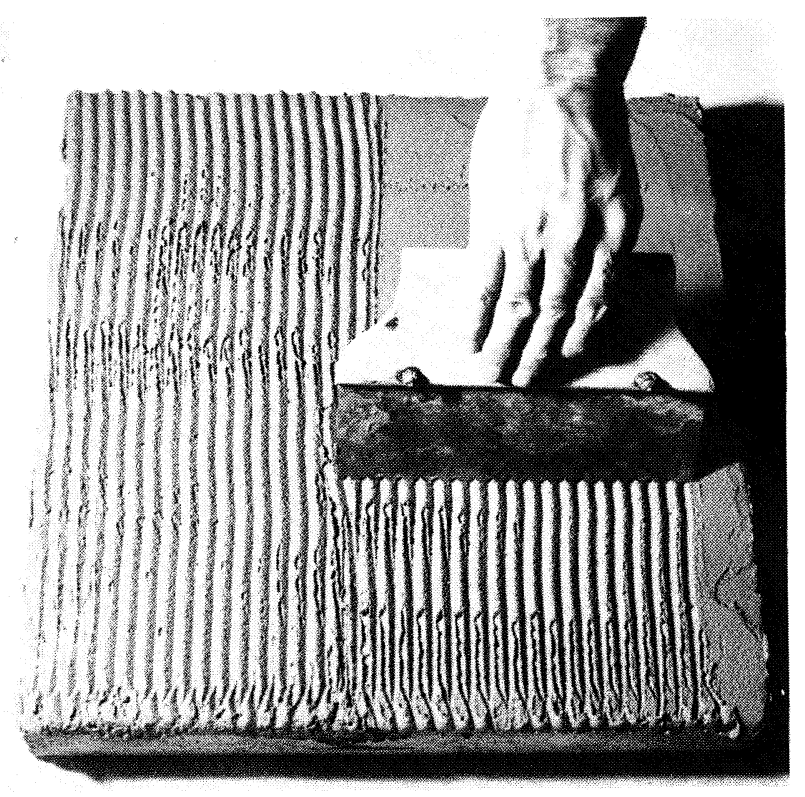

Preparación de una losa (que sirve como soporte) para efectuar los ensayos del mortero cola. Momento del peinado de la pasta.

absorber tensiones normales o tangenciales a la superficie de contacto mortero-soporte.

Las propiedades finales de la unión pieza cerámica/soporte, por medio de un mortero cola, dependen, no sólo de las características del material de agarre, sino también de las del soporte y de las de la propia operación de puesta en obra.

Esto no quiere decir que el mortero cola no pueda ser utilizado sin la preparación previa del soporte y con una puesta en obra poco cuidada; sino que la preparación del soporte, en caso necesario, aumenta notablemente el valor de la adherencia alcanzada la cual se incrementa igualmente con una aplicación cuidada.

A continuación se analizan brevemente las características de los tres factores mencionados que intervienen en la unión adhesiva.

\subsection{Características del mortero cola (adhesivo)}

\subsubsection{Compatibilidad con el soporte}

En primer lugar, el adhesivo debe ser compatible con el soporte. Una reacción indeseable entre ambos materiales perjudica gravemente su unión.

Los morteros cola son compatibles con la mayoría de los materiales que se emplean en la edificación como soportes donde se colocan los acabados de piezas cerámicas, excepto con el yeso, en particular cuando este material contiene un elevado grado de humedad.

Cuando se coloca un alicatado sobre un soporte de yeso, por medio de un mortero cola (de endurecimiento hidráulico), es aconsejable aislar el yeso del material de agarre por medio de una imprimación. para prevenir la probable reacción entre ambos materiales, en presencia de humedad, que daría lugar a la formación de ettringita (compuesto expansivo) 
y cuyo resultado previsible sería el desprendimiento del alicatado. La aplicación de la imprimación sobre el yeso debe hacerse cuando éste está seco (humedad inferior al $3 \%$ ); porque, en caso contrario, la imprimación sería rechazada por difusión del vapor de agua del soporte.

\subsubsection{Extendido inicial del producto}

Durante la aplicación, la pasta de mortero cola debe estar en un estado permanentemente deformable, que permita un extendido fácil sobre el soporte (sustrato), con lo que se consigue un íntimo contacto entre ambos materiales para facilitar su unión.

La mayor o menor facilidad en el extendido inicial de la pasta depende de sus características reológicas, propiciando un extendido óptimo, una baja tensión superficial de la fase líquida de la pasta, una suficiente cohesión de sus partes integrantes y una baja viscosidad de la misma.

La adherencia inicial (pegajosidad) del mortero cola depende, entre otros factores, de una viscosidad alta de la pasta, por lo que el valor óptimo que debe alcanzar este parámetro representa una solución de compromiso entre el que proporciona una adecuada adherencia inicial del material, pero al mismo tiempo permite un extendido correcto del mismo sobre el soporte.

La sujeción inicial de las piezas sobre una pared vertical se consigue cuando el umbral de cizallamiento de la pasta es mayor que el peso de la misma; en caso contrario, se produciría su deslizamiento y caída.

\subsubsection{Mojado del sustrato}

La pasta debe conseguir asimismo un mojado óptimo del soporte. El mojado de éste depende de las propiedades interfaciales; un valor reducido de la tensión superficial de la pasta favorece el mojado de aquél.

\subsubsection{Espesor de la capa de mortero}

El mortero cola se aplica en capa fina, con lo que se consiguen valores máximos de la fuerza de adherencia y de rigidez de la unión formada.

Se debe conseguir, asimismo, que la cantidad de pasta aplicada sea la suficiente para poder establecer el contacto necesario entre las dos superficies que forman la unión, procurando al mismo tiempo que la capa de pasta sea lo más uniforme posible.

\subsubsection{Fraguado y endurecimiento}

El material debe poseer, asimismo, un tiempo de fraguado y endurecimiento: ni demasiado rápido con lo que se disminuirían los rendimientos de aplicación y se dificultaría la ejecución del revestimiento, ni excesivamente lentos, como para tener que aplazar la puesta en servicio de éste fuera de un plazo razonable, que permita una puesta en obra correcta.

\subsubsection{Estabilidad dimensional}

La calidad de la unión adhesiva depende, asimismo, de la estabilidad dimensional del material.

Por otro lado, también puede influir en el comportamiento final el coeficiente de dilatación potencial frente a la humedad de las piezas cerámicas. Si éste es muy elevado pueden producirse tensiones importantes de cizallamiento en el plano de encolado. Sin embargo, una de las ventajas del encolado por capa fina con mortero cola es no tener que humedecer las piezas, gracias a su retención de agua, con lo cual se reducen notablemente las posibilidades de absorción de agua de la cerámica.

La pasta endurecida de mortero cola es sensible a la acción de la humedad, que produce un hinchamiento del material y una reducción de sus propiedades adhesivas. La magnitud de este hinchamiento depende de la formulación del material.

Sin embargo, el secado posterior de la pasta origina la recuperación, prácticamente total, de las características adhesivas primitivas del material (véase lo indicado en el Apartado 4).

\subsection{Características del soporte}

El soporte debe poseer las características que posteriormente se mencionan para que la unión adhesiva tenga la máxima efectividad. Estas características pueden ser mejoradas, en caso necesario, con la oportuna preparación del soporte.

\subsubsection{Cohesión}

El soporte debe poseer una cohesión y estabilidad mecánica suficientes para permitir la firme adherencia sobre él de las piezas a colocar, debiendo estar libre de partículas sueltas, polvo, grasa, restos de desencofrante, etc., que impidan una buena unión adhesiva.

\subsubsection{Propiedades superficiales}

La superficie del soporte debe ser algo áspera, mejor que demasiado lisa, con cuya característica se facilita un mayor contacto y enclavamiento del mortero cola sobre el soporte y una mejor unión adhesiva, a pesar de que ésta se produce no sólo por adherencia mecánica sino también por adherencia específica. Sin embargo, cuando la superficie es demasiado rugosa, es conveniente alisarla algo, para permitir un contacto más íntimo con el adhesivo y un flujo más suave de ésta al ser extendido por la superficie del sustrato durante su aplicación.

Por la misma razón, cuando el soporte presenta poros u oquedades de gran tamaño es necesario taponar éstos.

Estas operaciones suelen hacerse recubriendo el soporte con el propio mortero-cola cargado con arena.

\subsubsection{Porosidad}

El sustrato debe ser poroso o lleno de capilares abiertos, por donde el adhesivo pueda mojar o penetrar en la masa del soporte, fluyendo a través de ellos. 
La profundidad de la penetración por los capilares cilíndricos abiertos es, de acuerdo con la ley de Poiseuille, igual a

$$
d=\sqrt{\frac{\mathrm{A} \cdot \mathrm{r} \cdot \mathrm{t}}{2 \eta}}
$$

Donde: d profundidad de la penetración.

$\mathrm{t}$ tiempo de flujo.

$\eta$ viscosidad de la fase líquida del material de agarre.

A tensión de adhesión.

$r$ radio del capilar.

\subsection{Características de la puesta en obra y condiciones ambientales durante la misma}

Por último, la calidad de la unión adhesiva depende también de la forma y condiciones de la puesta en obra (colocación).

\subsubsection{Extendido}

Generalmente, el extendido de la pasta ya preparada sobre el soporte a revestir se realiza a llana y después se peina con la espátula dentada para uniformar el espesor. La superficie máxima de pasta extendida sobre el soporte, de una sola vez, es función del tiempo abierto, de la velocidad de formación de película y naturalmente de las condiciones ambientales (ver Apartado 6.3.3.), de tal manera que puedan aplicarse las piezas con garantía de que la adherencia final sea como mínimo la especificada de $5 \mathrm{kp} / \mathrm{cm}^{2}$.

\section{En este sentido hay que señalar el peligro que para la permanencia del revestimiento tienen los sistemas de contratación de la puesta en obra por destajo, en los que, para ganar «tiempo», el operario extiende el mortero en gran superficie, sin tener en cuenta que el tiempo transcurrido desde la colocación de la primera pieza hasta la última pueda ser superior al tiempo útil, o sea al tiempo abierto del mortero cola.}

Cuando esto se produce, aunque la pieza pueda quedar adherida inicialmente, al cabo de cierto tiempo termina desprendiéndose apareciendo limpia de mortero su cara de agarre.

\subsubsection{Presión}

Una colocación óptima de las piezas cerámicas con un mortero cola debe hacerse:

- fijando éstas con un ligero movimiento de rotación, para romper eventualmente la película superficial que pueda formarse sobre la capa de mortero cola;

- imprimiendo sobre la plaqueta una presión adecuada que permita que el exceso de material de agarre fluya fuera de ésta, con el fin de lograr un contacto perfecto sobre la superficie de unión; y

- en superficies verticales manteniendo esta presión durante el tiempo imprescindible para asegurar que la resistencia inicial de la unión supera las tensiones especificas inherentes a esta forma de encolado.

\subsubsection{Condiciones ambientales}

Las condiciones ambientales existentes en el momento de la puesta en obra y en las etapas posteriores a ésta, pueden influir desfavorablemente, en la unión adhesiva, si tales condiciones son adversas, de dos maneras distintas: favoreciendo la evaporación rápida del agua de amasado del material de agarre y provocando movimientos en el soporte.

La evaporación del agua de amasado se favorece con temperaturas y velocidades de circulación del aire altas durante la colocación de los revestimientos y pavimentos cerámicos.

La eliminación parcial del agua de amasado produce en el material de agarre una reducción de sus características mecánicas de adherencia y acelera asimismo el fraguado del material.

Por su parte, cuando el soporte - caso por ejemplo de tratarse de una fachada- está sometido a diferencia de temperaturas importantes a lo largo del día, pueden originarse en él contracciones y dilataciones que favorecen el despegue y caída de los revestimientos cerámicos colocados sobre éste.

Ambos fenómenos tienen una marcada importancia en la colocación de materiales cerámicos con morteros de cemento tradicionales.

Cuando el material de agarre es un mortero cola la importancia de estos fenómenos es menos acusada, porque este material posee unas características intrínsecas que le permiten contrarrestar en parte estos hechos.

En primer lugar, el mortero cola presenta un cierto poder de retención de agua, cuyo grado depende de la formulación del material y, asimismo, el producto posee una elasticidad (alargamiento a la rotura) mayor que el mortero tradicional, que le permite, hasta cierto grado, absorber pequeñas variaciones dimensionales.

\section{Características del mortero cola}

Hoy en día están universalmente admitidas las características de los morteros cola según la definición establecida por la Unión Europea para la apreciación técnica de materiales y procedimientos de construcción no tradicionales (U.E.A.t.c.).

Este organismo internacional define para el mortero cola tres tipos de características: de identificación, trabajabilidad y durabilidad, que se determinan por medio de los oportunos ensayos.

\subsection{Identificación}

Las caracteristicas de identificación tienen por objeto únicamente comprobar que el producto conserva una constante en sus propiedades, de una partida a otra, a lo largo de su fabricación.

Los parámetros experimentales elegidos para conocer la regularidad del producto son: la masa volúmica aparente, la pérdida al fuego a $4500^{\circ} \mathrm{C}$ y la granulometría, medidos sobre el producto en polvo; y la masa volúmica, el poder de retención de agua y el tiempo abierto teórico, sobre la pasta. 
Además de ser una caracteristica que sirve para definir la constancia de calidad del producto, a éste se le exige un poder de retención de agua tal que, en las condiciones del ensayo, la pérdida de agua de la pasta sea inferior a $6 \mathrm{~g}$.

\subsection{Trabajabilidad}

$\mathrm{Al}$ mortero cola se le exige en cuanto a trabajabilidad las siguientes características:

- un tiempo abierto, superior a $20 \mathrm{~min}$.

- un tiempo de ajuste manual, superior a $10 \mathrm{~min}$.

- un tiempo de vida de la mezcla en el bote, superior a $3 \mathrm{~h}$.

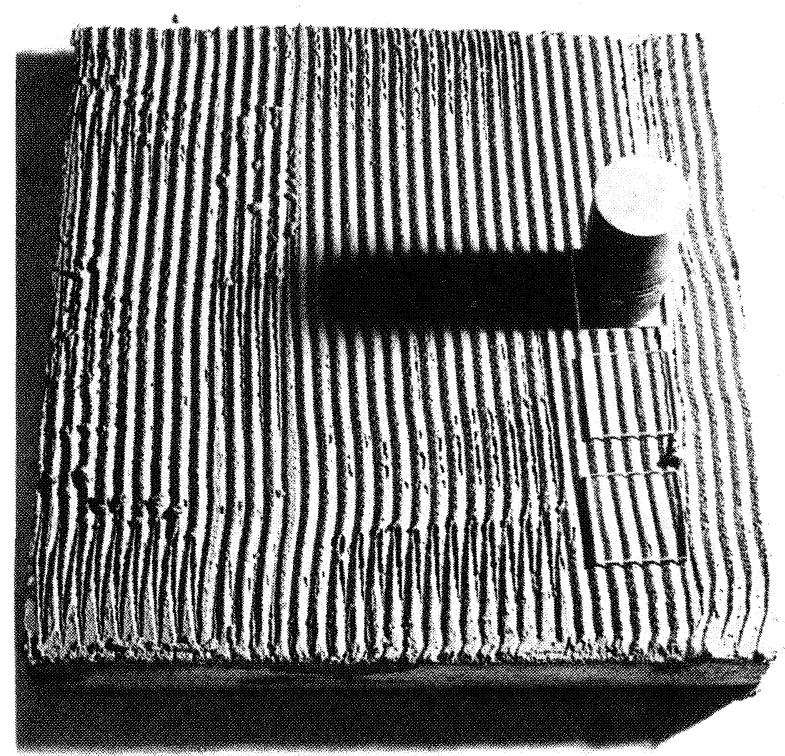

Ensayo de "transferencia». 1." Secuencia. Colocación sobre la pasta de mortero cola peinada de tres piezas de vidrio de $5 \times 5 \mathrm{~cm}$ y aplicación sobre una de ellas de una carga de $0,5 \mathrm{~kg}\left(0,02 \mathrm{~kg} / \mathrm{cm}^{2}\right)$ durante $30 \mathrm{seg}$

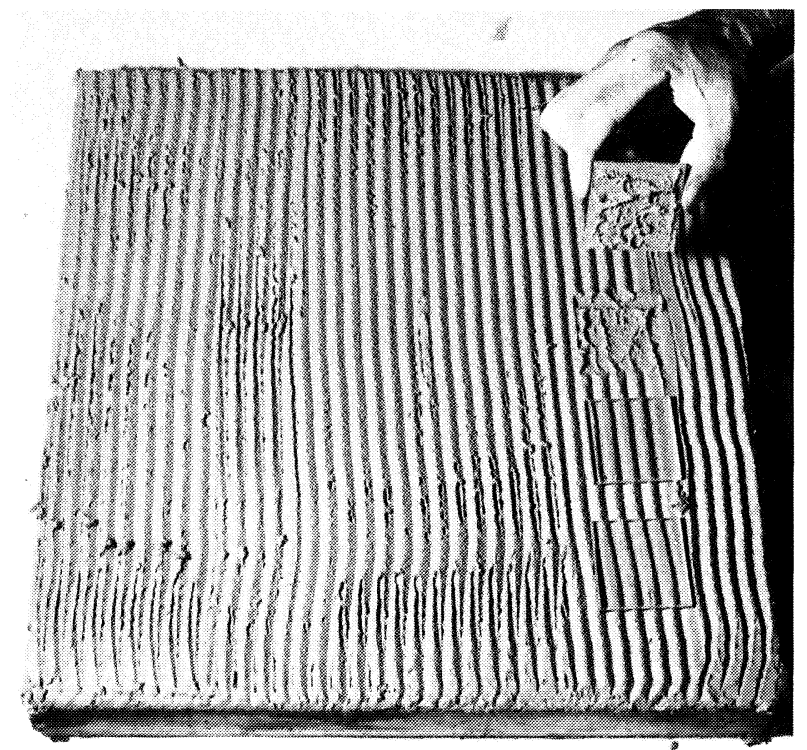

Ensayo de "transferencia». 2." Secuencia. Arrancamiento manual de la pieza de vidrio anterior. En el vidrio puede apreciarse la zona de su superficie con pasta transferida.
- un anclaje inicial (pegajosidad) de las piezas cerámicas (colocadas con el producto) tal que no se produzca un deslizamiento de las mismas sobre una pared vertical superior a $2 \mathrm{~mm}$, medido en las primeras 24 horas.

- una deformación y mojabilidad de la pasta tal que permita una «transferencia» de ella superior al $65 \%$.

\subsection{Durabilidad}

Por último, al producto se le exige una durabilidad (o conservación de la calidad) adecuada frente a la acción de las distintas solicitaciones que pueden darse en la edificación.

\section{La «transferencia» se determina separando, de la capa de mortero fresca, varias piezas de vidrio colocadas sobre ella (a los 20 minutos de extendida y peinada). Cada pieza de vidrio se mantiene, antes de su arrancamiento, cargada durante 30 segundos con un peso de $0,02 \mathrm{~kg} / \mathrm{cm}^{2}$. El porcentaje medio de plaqueta de vidrio con restos de pasta adherida en su superficie, es el número que mide la «transferencia».}

Estas exigencias son distintas según sea el empleo a que puede ser destinado el material: si para la colocación de revestimientos murales o de suelos; siendo diferentes también estas exigencias si la utilización va a ser efectuada en el interior o en el exterior del edificio.

En primer lugar, al material, en todos los casos, se le exige una calidad inicial mínima, medida por la fuerza de adherencia después de un curado en seco, en condiciones normales, de 28 días de duración. Eśta adherencia deberá ser igual o superior a $5 \mathrm{~kg} / \mathrm{cm}^{2}$.

Las solicitaciones consideradas con carácter general (es decir, para revestimientos de paredes y suelos, en el interior y exterior), son las relativas a la acción del:

- calor $\left(65^{\circ} \mathrm{C}\right), \mathrm{y}$

- humedad;

$\mathrm{y}$, especificamente: para revestimiento de suelos interiores

- comportamiento al choque;

y para revestimientos murales exteriores

- hielo deshielo.

Asimismo, eventualmente, para soportes distintos del hormigón, se determina el comportamiento frente a la acción de

- la humedad (que asciende por capilaridad a través de la superficie del soporte opuesta a la que lleva colocado el mortero de agarre).

La adherencia final del material, medida después de ser sometido a los tratamientos o solicitaciones antes mencionados, debe ser igual o superior a $5 \mathrm{~kg} / \mathrm{cm}^{2}$.

Este valor minimo de la adherencia. medido por la fuerza de arrancamiento, corresponde al que se exige en los ensayos en que las piezas, sujetas con el material de agarre a ensayar, se colocan sin un sellado en sus 
juntas siendo de $10 \mathrm{~kg} / \mathrm{cm}^{2}$ el valor mínimo que se establece para aquellos otros en que las piezas llevan las juntas selladas.

\section{Modo de medir estas características}

Todas las caracteristicas antes indicadas se determinan, según el método experimental establecido en las Directrices de la U.E.A.t.c., por medio de un ensayo destructivo de medida de la fuerza de tracción vertical necesaria para el arrancamiento de piezas cerámicas adheridas con el material de agarre (mortero cola) sobre un soporte.

En resumen, la técnica experimental seguida en el ensayo de arrancamiento indicado, es la que se describe a continuación:

En primer lugar, el producto en polvo se mezcla con la proporción de agua indicada por el fabricante, hasta obtener una pasta homogénea que se deja en reposo (en el recipiente donde se ha realizado la mezcla) el lapso de tiempo recomendado por éste - del orden de 15 ó 20 min.-.

A continuación, la pasta se extiende y peina (con una espátula dentada) sobre el soporte, formado por una losa de hormigón de $60 \times 60 \mathrm{~cm}$ y $4 \mathrm{~cm}$ de espesor.

$\mathrm{Al}$ cabo de un lapso de tiempo de 10 ó 15 min. después de extendida la pasta, se colocan sobre ella 9 piezas de cerámica porosa o de gres, según el tipo de ensayo, de dimensiones $5 \times 5 \mathrm{~cm}$, separadas entre sí y de los bordes de la losa por espacios iguales. Estas piezas quedan sujetas por medio de la presión ejercida por una carga de $2 \mathrm{~kg}$, que se coloca y mantiene sobre cada una de ellas durante $30 \mathrm{seg}$.

En el ensayo de Tiempo abierto, este lapso de tiempo es también de 20 y 30 min.

En las condiciones del ensayo la película formada en la superficie de la pasta de mortero cola no se elimina, por lo que los valores de adherencia obtenidos son inferiores a los que resultan en una aplicación que tuviera en cuenta las precauciones señaladas en el Apartado 6.3.2.

Tales precauciones no están recogidas en los métodos de ensayo anteriormente indicados, porque éstos tienen por finalidad: la obtención de unos valores de adherencia más acordes con los que resultan en una puesta en obra de tipo industrializado - ya mencionada -, y la reproducibilidad de resultados que se vería reducida por el hecho de introducir en el ensayo una nueva operación manual.

Después del curado de la pasta de mortero cola en las condiciones especificadas en cada ensayo - cuya duración es, en general, de 28 días-, se colocan unas tes de hierro sobre las piezas cerámicas que están sujetas al soporte por medio del material de agarre a ensayar. Las tes quedan sólidamente unidas a las plaquetas por medio de un adhesivo de gran potencia (resina epoxi).

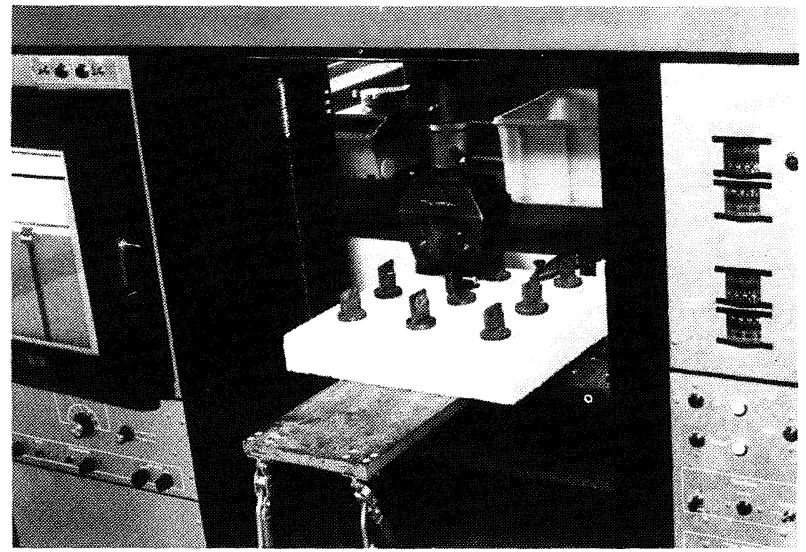

Ensayo de adherencia de un mortero cola. Momento de realizar la prueba de arrancamiento por medio de una máquina de tracción. En la fotografia puede apreciarse la losa con las piezas cerámicas de $5 \times 5 \mathrm{~cm}$ adheridas puede apreciarse la losa con las piezas cerámicas de $5 \times 5 \mathrm{~cm}$ adheridas
con el mortero cola a ensayar y sobre las que se han colocado las correspondientes tes metálicas.

El valor de adherencia del mortero cola se obtiene, en cada caso, por medida de la fuerza de arrancamiento (tracción vertical) en una máquina de tracción hidráulica o neumática, cuya fuerza se aplica sobre las tes de hierro (que se sujetan a la máquina por medio de una mordaza) a razón de $10 \mathrm{~kg} / \mathrm{seg}$

\section{Bibliografía}

(1) Directrices UEAtc pour l'Agrément des colles pour revêrements céramiques.

(2) Taylor, H.F. The Chemistry of Cements. Academic Press (1964).

(3) Arthur K. Doolitte, The Technology of Solvents and Plasticizers. Ed. Wiley. New York (1957).

(4) Inving Skeik, Manual de adhesivos. Cía. Ed. Continental, Sociedad Anónima (1966).

(5) Bombled, J.P., Comportement rhéologique des pâtes mortiers et bétons. Revue des Matériaux de Construction n. ${ }^{\circ} 617$, febrero (1967).

(6) Brocard, M.J., Utilisation des matières plastiques dans le gros oeuvres. Annales de l'ITBTP. Dec. (1960)

(7) Hosek, J., Properties of Cement Mortars Modified by Polymer Emulsion. J. ACI Dec. (1966).

(8) Stamatia, A., Frosdistou-Yannes and Surendue P. Shan Polymer latex modified mortar. J. ACI En. (1972)

(9) Adamson, A., Physical Chemistry of Surfaces. Intrescience Publishers. New York (1966)

(10) Alvarez Estrada, D., Aplicación de revestimientos cerámicos en exteriores. Su problemática y solución. Monografía n. ${ }^{\circ} 301$ del IETcc (1972).

(11) Valdehita, M.T., Morteros de cemento para albañilería. Monografía n. ${ }^{\circ} 337$ del IETcc (1976).

(12) Olmo, C. del, Reología de pastas de cemento modificadas. (Trabajo en preparación). 